\title{
Mini Review on: Simple, Practical, and Evidence-Based - an Algorithm for The Diagnosis and Treatment of Iron Deficiency
}

\author{
Erland Erdmann*
}

University in Cologne, Germany

Article Info

\section{Article Notes}

Received: November 11, 2018

Accepted: November 28, 2018

\section{*Correspondence:}

Prof. Dr. med. Erland Erdmann, University in Cologne, Germany; Telephone No: +49 221 487487; Fax No: +49 221 2824748; Email: erland.erdmann@uni-koeln.de.

(c) 2018 Erdmann $\mathrm{E}$. This article is distributed under the terms of the Creative Commons Attribution 4.0 International License.

\section{Keywords}

Chronic heart failure (CHF)

Iron deficiency

Ferritin

Transferrin saturation (TSAT)

Intravenous iron therapy

\section{Abstract}

Iron deficiency is a common comorbidity in chronic heart failure (CHF) with a negative impact on the outcome of the patient. Even without anaemia iron deficiency can cause decreased physical performance, poor quality of life, and increased mortality. Therefore it is important to diagnose and treat iron deficiency, especially in patients with CHF.

Despite the great relevance for the outcome of CHF-patients little attention is being payed to iron deficiency in these patients, even though a specific diagnosis and therapy is available and is recommended by the current ESC guidelines.

A simple, practical and evidence based algorithm is available and can now help to improve diagnosis and treatment of iron deficiency in everyday practice. This article reviews a discussion of a group of German cardiologists who aimed to give practical advice on how to diagnose and treat iron deficiency in $\mathrm{CHF}$ patients.

Increased life expectancy and improved acute treatment of cardiovascular diseases have led to a continuously increasing prevalence of heart failure. There are an estimated 1.8 million people suffering from chronic heart failure (CHF) in Germany (in 2010) and up to 300.000 additional new patients per year ${ }^{1}$. The number of hospitalisations for CHF is also increasing ${ }^{2}$.

Frequently, systemic iron deficiency is a concomitant comorbidity of heart failure occurring in up to $50 \%$ of the CHF patients ${ }^{3}$. Numerous abnormalities can lead to iron deficiency in CHF patients: these include malnutrition or an unbalanced diet (e.g. in CKD patients), decreased intestinal iron adsorption due to intestinal wall abnormalities, and concomitant medications $\mathrm{s}^{4}$.

In addition to disturbing erythropoiesis, iron deficiency also impairs the function of numerous iron-containing enzymes involved in the oxidative metabolism (e.g. cytochromes), the mitochondrial respiratory chain, as well as the cellular energy balance ${ }^{5,6}$. For this reason, iron deficiency may lead to decreased muscular function and an associated, reduced, general performance even at a time clearly preceding the development of manifest anaemia ${ }^{7}$. Besides, study data demonstrate that in CHF patients, iron deficiency results in symptoms being subjectively perceived as worse (as measured by the NYHA classification) ${ }^{3}$, in an impaired quality of life $^{8}$, and in a reduced physical performance ${ }^{9}$ - irrespective of the anaemia status. Its negative influence on morbidity and mortality in patients with impaired pumping function (HFrEF) makes concomitant iron deficiency an independent prognostic factor for a poorer outcome ${ }^{7}$. 


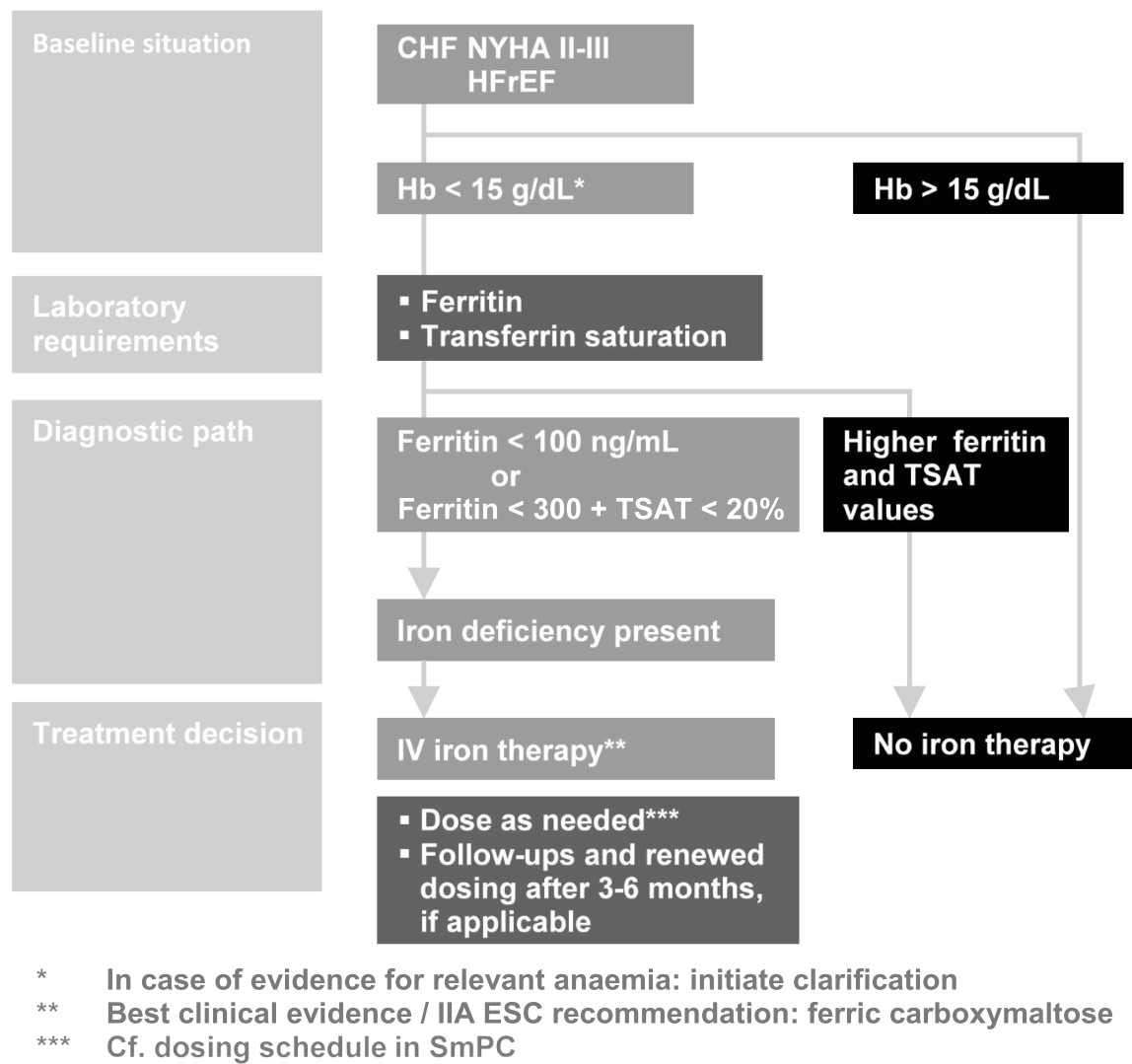

Figure 1: Algorithm for diagnosis and treatment of iron deficiency in patients with CHF, adapted from (13).

Abbreviations: CHF: chronic heart failure; HFrEF: heart failure with reduced ejection fraction; Hb: haemoglobin; TSAT: transferrin saturation; IV: intravenous; ESC: European Society of Cardiology, SmPC: summary of product characteristics

On the other hand, studies showed that treatment with intravenous ferric carboxymaltose (FCM) improves physical performance and NYHA class, and that it increases the quality of life and decreases the hospitalisation rate ${ }^{10,11}$.

Currently however, the diagnosis and treatment of iron deficiency are still too seldom in everyday practice ${ }^{12}$ with the consequence that IV iron therapy is not available to many patients who might benefit from it. In order to change this, 9 leading German cardiologists convened and discussed ways to place more attention on this clinically relevant comorbidity in everyday practice ${ }^{13}$. The result is this review that aims to give practical advice on the diagnosis and treatment of iron deficiency in CHF patients.

The experts agreed that iron deficiency must be considered when seeing a CHF-patient, adequately diagnosed and then treated. An algorithm for diagnosis and treatment, that is already available (Fig.1) and is consistent with the contents of the European Society of Cardiology's (ESC) guidelines on the diagnosis and treatment of acute and chronic heart failure, was generally deemed as useful. This algorithm should be regularly included in the clinical routine for CHF patients to ensure adequate diagnosis and a resulting effective treatment ${ }^{13}$.
Haemoglobin and serum iron values are unsuitable as parameters for diagnosing iron deficiency as approx. one third of the iron-deficient CHF patients presents without concomitant anaemia ${ }^{14}$. In the experts' opinion, the $\mathrm{Hb}$ value merely serves as an instrument to clarify any additional presence of anaemia. The serum iron level shows individual and circadian fluctuations and is highly dependent on food intake, rendering it impossible to make a reliable statement on the patient's iron status using serum iron measurements ${ }^{15}$.

A safe and practicable way to demonstrate iron deficiency is the measurement of serum ferritin level and transferrin saturation (TSAT) as recommended in the ESC guidelines on the diagnosis of iron deficiency (evidence level $\mathrm{C}$, recommendation level $\mathrm{I}{ }^{16}$. Iron deficiency requiring treatment is given at a ferritin level $<100 \mathrm{ng} /$ $\mathrm{mL}$ or between $100-300 \mathrm{ng} / \mathrm{mL}$ if concomitantly TSAT is $<20 \%$. According to the experts, these parameters should be a constituent part of the routine diagnostic measures in CHF patients.

As far as the treatment of iron deficiency is concerned the expert opinion was consistent with the ESC guidelines: if the patient presents with iron deficiency, this deficiency should be corrected effectively. During the expert meeting, the use 
of different iron preparations was discussed, including the advantages and disadvantages of oral and intravenous preparations. Oral iron therapy is not recommended for CHF patients. The background for this are the results of the IRONOUT study where oral iron treatment did not lead to an improvement in performance (as measured by spiroergometry) and where replenishment of iron stores was also only marginal as compared to placebo ${ }^{17}$.

Moreover, studies with intravenous ferric carboxymaltose (FCM) were assessed. In contrast to oral iron therapy, the administration of intravenous FCM improved the patients' functional performance parameters, NYHA class, and the quality of life in iron-deficient CHF patients ${ }^{10,11,18}$. Not the least, IV iron therapy with FCM was able to reduce the hospitalisation rate ${ }^{19}$.

No data are available on the IV administration of other iron formulations in CHF patients.

Therefore, the ESC also recommends considering intravenous treatment with ferric carboxymaltose in symptomatic patients with $\mathrm{CHF}$ and impaired left ventricular ejection fraction (LVEF) as well as iron deficiency (independent of the presence of anaemia) to improve heart failure symptoms, performance, and quality of life (evidence level A, recommendation level IIa) ${ }^{16}$.

The dosage for substitution using FCM can be established easily on the basis of body weight and baseline haemoglobin value ${ }^{13}$, with several clinical studies showing that in CHF patients the iron deficit ranges from 1000 to $1800 \mathrm{mg}^{10,11,20,21,22}$.

The treatment response should be followed in accordance with the discussed algorithm after three months (in case of an initial administration of $500 \mathrm{mg}$ iron) or six months (in case of an initial administration of $1000 \mathrm{mg}$ iron or more [with repeated dosing]). If ferritin and TSAT laboratory values again fall below the thresholds specified above, renewed intravenous iron substitution should be considered $^{13}$. The experts of this meeting followed this assessment $^{23}$.

To avoid iron overload, it is not recommended to start an intravenous iron therapy if the laboratory values of ferritin and TSAT are higher than the above-mentioned cut-off values. Iron excess accumulates in cells, and in higher concentrations causes oxidative stress and triggers cardiomyocytes necrosis ${ }^{24}$. It seems that an increased ferritin concentration is associated with an increased risk of atrial fibrillation in the general population ${ }^{25}$. Therefore it is necessary, especially in case of repeated doses, to reassess iron parameters before each iron substitution. To minimize the risk of Non-transferrin bound serum iron (NTBI), and therefore acute toxicities, it is also important to comply with the specification of iron preparations (e.g. dilution, maximum dose and infusion time).
Also it is mandatory to monitor patients for at least 30 minutes after an intravenous iron preparation and to have the appropriate life support care on hand for handling rare but serious allergic reactions, which are even possible with new iv iron formulations.

Provided the respective logistic prerequisites (space, observation) are readily available, intravenous iron therapy may well be performed at the general physician's office. If this is not possible, the experts recommend that iron substitution be performed in a hospital, under daycare conditions, or at an office-based cardiologist's ${ }^{23}$.

\section{Conclusion}

Iron deficiency is an underestimated but serious and common comorbidity of CHF that negatively affects symptoms, quality of life, hospitalisation rate and mortality 3,7,10,19. Despite numerous pieces of evidence demonstrating that intravenous iron substitution has a positive effect on the patients' symptoms and outcomes, register studies show that concomitant iron deficiency is underdiagnosed and seldom adequately treated ${ }^{12}$. According to 9 leading German cardiologists at their expert meeting the published algorithm for diagnosis and treatment of iron deficiency ${ }^{13}$ can easily and practically be integrated in the clinical routine treatment of CHF patients, thus resulting in improved patient care $^{23}$.

\section{Conflict of interest}

Erland Erdmann within the last 5 years has received travel grants and speaker's honoraria from Bayer, Roche, Novartis and Vifor.

This work was supported by an unrestricted grant by Vifor Pharma Deutschland GmbH.

\section{References}

1. Zugck C, Müller A, Helms TM. et al. Gesundheitsökonomische Bedeutung der Herzinsuffizienz: Analyse bundesweiter Daten. Dtsch Med Wochenschr. 2010; 135: 633-638.

2. Christ M, Störk S, Dörr M, et al. Heart failure epidemiology 200-2013: insights from the German Federal Health Monitoring System. Eur J Heart Fail. 2016; 18: 1009-1018.

3. Klip IT, Comin-Colet J, Voors AA, et al. Iron deficiency in chronic heart failure: an international pooled analysis. Am Heart J. 2013; 165(4): 575-582.

4. McDonagh T, Macdoughall IC. Iron therapy for the treatment of iron deficiency in chronic heart failure: intravenous or oral. Eur J Heart Fail. 2015; 17: 248-262.

5. Haddad S, Wang Y, Galy B, et al. Iron-regulatory proteins secure iron availability in cardiomyocytes to prevent heart failure. Eur Herat J. 2017; 38(5): 362-372.

6. Hoes MF, Beverborg NG, Kijlstra JD, et al. Iron deficiency impairs contractility of human cardiomyocytes through decreased mitochondrial function. Eur J Heart Fail. 2018; 20: 910-919.

7. Jankowska EA, Rozentryt P, Witkowska A, et al. Iron deficiency: an ominous sign in patients with systolic chronic heart failure. Eur Heart J. 2010; 31: 1872-1880. 
8. Comin-Colet J, Enjuanes C, González G, et al. Iron deficiency is a key determinant of health-related quality of life in patients with chronic heart failure regardless of anaemia status. Eur J Heart Fail. 2013:15(10): 1164-72.

9. Martens P, Nijst P, Verbrugge FH, et al. Impact of iron deficiency on exercise capacity and outcome in heart failure with reduced, midrange and preserved ejection fraction. Acta Cardiol. 2018; 73(2): 115123.

10. Anker SD, Comin-Colet J, Filippatos G, et al. Ferric carboxymaltose in patients with heart failure and iron deficiency. N Engl J Med. 2009 361: 2436-2448.

11. Ponikowski P, van Veldhuisen DJ, Comin-Colet J, et al. CONFIRM-HF investigators: Benefical effects of long-term intravenous iron therapy with ferric carboxymaltose in patients with symptomatic heart failure and iron deficiency. Eur Heart J. 2015; 36(11): 657-668.

12. Wienbergen $\mathrm{H}$, Pfister $\mathrm{O}$, Hochadel $\mathrm{M}$, et al. Usefulness of Iron Deficiency Correction in management of Patients With Heart Failure [from the Registry Analysis of Iron Deficiency-Heart Failure (RAIDHF) Registry]. Am J Cardiol. 2016 Dec 15; 118(12): 1875-1880.

13. Doehner W, Blankenberg S, Erdmann E, etal. Eisenmangel bei Patienten mit chronischer Herzinsuffizienz - diagnostische Algorithmen und therapeutische Optionen anhand der aktuellen Studienlage. Dtsch Med Wochenschr. 2017; 142(10): 752-757.

14. von Haehling S, Gremmler U, Krumm M, et al. Prevalence and clinical impact of iron deficiency and anaemia among outpatients with chronic heart failure: The PrEP Registry. Clin Res Cardiol. 2017; 106(6): 436-443.

15. Herklotz H, Huber A. Labordiagnose von Eisenstoffwechselstörungen. Schweiz Med Forum. 2010; 10 (30-31): 500-507.

16. Ponikowski P, Voors AA, Anker SD, et al. 2016 ESC Guidelines for the diagnosis and treatment of acute and chronic heart failure. Eur J Fail. 2016; 18(8): 891-975.

17. Lewis GD, Malhotra R, Hernandez AF, et al. Effect of oral iron repletion on exercise capacity in patients with heart failure with reduced ejection fraction and iron deficiency: The IRONOUT HF Randomized Clinical Trial. JAMA. 2017; 317(19): 1958-1966.

18. Anker SD, Kirwan BA, van Veldhuisen DJ, et al. Effects of ferric carboxymaltose on hospitalisations and mortality rates in irondeficient heart failure patients: an individual patient data metaanalysis. Eur J Heart Fail. 2018; 20(1): 125-133.

19. Jankowska EA, Tkaczyszyn M, Suchocki T, et al; Fair HF Trial Investigators. Effects of intravenous iron therapy in iron-deficient patients with systolic heart failure: a meta-analysis of randomized controlled trials. Eur J Heart Fail. 2016; 18(7): 786-795.

20. Toblli JE, Lombraña A, Duarte P, et al. Intravenous iron reduces NTpro-brain natriuretic peptide in anemic patients with chronic heart failure and renal insufficiency. J Am Coll Cardiol. 2007; 50(17): 16571665.

21. Okonko DO, Grzeslo A, Witkowski T, et al. Effect of intravenous iron sucrose on exercise tolerance in anemic and nonanemic patients with symptomatic chronic heart failure and iron deficiency FERRIC-HF: a randomized, controlled, observer-blinded trial. J Am Coll Cardiol. 2008; 51(2): 103-112.

22. Beck-da-Silva L, Piardi D, Soder S, et al. IRON-HF study: a randomized trial to assess the effects of iron in heart failure patients with anemia. Int J Cardiol. 2013; 168(4): 3439-3442.

23. Erdmann E, Bauersachs J, Doehner W, et al. Eisenmangel bei Herzinsuffizienz - neuer Diagnose-Therapie-Algorithmus, evidenzgeprüft und praktikabel. MMW-Fortschr. Med. 2018; 160(S4): 8-11.

24. Wang $\mathrm{Y}, \mathrm{Wu} \mathrm{M}, \mathrm{Al}-$ Roussan $\mathrm{R}$, et al. Iron-induced cardiac damage: role of apoptosis and deferasirox intervention. J Pharmacol Exp Ther. 2011; 336: 56-63.

25. Mikkelsen LF, Nordestgaard BG, Schnohr P, et al. Increased Ferritin Concentration and Risk of Atrial Fibrillation and Heart Failure in Men and Women: Three Studies of the Danish General Population Including 35799 Individuals. Clin Chem. 2018 Nov 20. pii: clinchem.2018.292763. doi: 10.1373/clinchem.2018.292763. [Epub ahead of print]. 